\title{
Observations from a multicentre study on the use of the sputum specimen in patients hospitalized with community-acquired pneumonia
}

\author{
EL Taylor $M D^{1}$, TJ Marrie MD FRCPC ${ }^{1,2}$, MJ Fine $M D^{1}$, DS Obroskyl MSc ${ }^{3}, W N$ Kapoor $\mathrm{MD}^{3}$, \\ C Coley $\mathrm{MD}^{4}$, DE Singer $\mathrm{MD}^{4}$
}

\begin{abstract}
EL Taylor, TJ Marrie, MJ Fine, et al. Observations from a multicentre study on the use of the sputum specimen in patients hospitalized with community-acquired pneumonia. Can J Infect Dis 1999;10(1):39-47.

OBJECTIVE: To describe the use of sputum Gram stain and culture in patients with community-acquired pneumonia (CAP) and to determine the factors that are associated with obtaining sputum for culture.

TYPE OF STUDY: Prospective observational cohort study of patients hospitalized for treatment of CAP at four medical institutions in three geographic locations.

MAIN MEASUREMENTS: Results of Gram stain and culture of sputum; comparison of patients who had sputum processed for culture within $24 \mathrm{~h}$ of admission with those who did not have such a specimen processed during the first week of hospitalization; and the results of investigator assignment of etiology of pneumonia according to predefined criteria. RESULTS: Four hundred and seventy-eight of 1339 (36\%) patients had a sputum specimen processed for culture within $24 \mathrm{~h}$ of admission. Patients who had a sputum specimen processed within $24 \mathrm{~h}$ of admission were more likely to be hospitalized at the Boston site (odds ratio [OR] 20.6) or Pittsburgh sites (3.4) and to have current sputum production, chronic obstructive lung disease and moderate or large amount of sputum. Female sex (0.4), neutropenia (0.05), and do not resuscitate status (0.36) were important predictors of failure to have a sputum processed for culture. The rate of Streptococcus pneumoniae isolation was highest in Boston, 53 of $269(19.3 \%)$ patients $(\mathrm{P}<0.001)$ compared with the other sites; Moraxella catarrhalis was isolated only at the Boston site. Sputum culture results served as the basis for the assignment of an etiological diagnosis of the pneumonia by investigators in $67 \%$ of 397 patients. CONCLUSIONS: Sputum is not processed for culture in the majority of patients with CAP. The factors that determine whether sputum is processed for culture within $24 \mathrm{~h}$ of admission are site of care and a variety of patient factors. Common respiratory pathogens when present in sputum culture tend to be used to assign an etiological diagnosis. A positive sputum culture result appears not to result in a more favourable outcome.
\end{abstract}

Key Words: Diagnosis, Gram stain, Pneumonia, Sputum

Pour le résumé, voir page 40

\footnotetext{
${ }^{1}$ Departments of Medicine and ${ }^{2}$ Microbiology, Dalhousie University and the Victoria General Hospital, Halifax, Nova Scotia; ${ }^{3}$ Division of General Internal Medicine, Department of Medicine, University of Pittsburgh, Pittsburgh, Pennsylvania; ${ }^{4}$ General Internal Medicine Unit, Massachusetts General Hospital, Boston and Harvard University, Cambridge, Massachusetts

Correspondence and reprints: Dr Thomas J Marrie, 1278 Tower Road, Room 5014 ACC, Victoria General Hospital, Halifax, Nova Scotia B3H 2Y9. Telephone 902-473-5553, fax 902-473-7394, e-mail tmarrie@is.dal.ca

Received for publication February 2, 1998. Accepted June 30, 1998
} 


\title{
Observations tirées d'une étude multicentrique sur l'emploi des spécimens d'expectorations chez des patients hospitalisés pour pneumonie acquise dans la communauté
}

\begin{abstract}
OBJECTIF : Décrire l'emploi de la coloration de Gram et des cultures de spécimens d'expectorations chez des patients atteints de pneumonie acquise dans la communauté (PAC) et déterminer les facteurs associés à la cueillette des spécimens aux fins de cultures.

TYPE D'ÉTUDE : Étude d'observation prospective sur une cohorte de patients hospitalisés pour le traitement de la PAC dans quatre établissements de santé de trois villes distinctes.

PRINCIPAUX PARAMÈTRES : Résultats des colorations de Gram et des cultures d'expectorations : comparaison des patients qui ont subi leur prélèvement de spécimens pour culture dans les 24 heures suivant leur admission et de ceux qui n'ont pas subi ce test au cours de leur première semaine d'hospitalisation; et résultats de l'assignation de l'étiologie par les investigateurs en fonction de critères prédéfinis.

RÉSULTATS : Quatre cent soixante-dix-huit patients sur 1339 (36\%) ont subi une culture d'expectorations dans les 24 heures suivant leur hospitalisation. Ces patients étaient plus susceptibles d'être hospitalisés aux centres de recherche de Boston (risque relatif [RR] 20,6) ou de Pittsburgh (3,4) et d'expectorer encore, de souffrir d'une maladie pulmonaire obstructive chronique ou de produire une quantité moyenne à importante d'expectorations. Le fait d'être de sexe féminin $(0,4)$, la neutropénie $(0,05)$ et la mention au dossier "ne pas réanimer » $(0,36)$ ont été d'importants facteurs de prévisibilité que ne soit prélevé aucun spécimen d'expectorations à des fins de culture. Le taux de souches de Streptococcus pneumoniae isolées a été le plus élevé à Boston, 53 patients sur 269 (19,3\%; p < 0,001) en comparaison avec les autres sites; Moraxella catarrhalis a été isolé à Boston seulement. Les résultats des cultures d'expectorations ont servi de base à l'assignation d'un diagnostic étiologique de pneumonie par les investigateurs chez $67 \%$ des 397 patients.

CONCLUSIONS : Les expectorations ne sont pas mises en culture chez la majorité des patients atteints de PAC. Les facteurs qui font que l'on procède ou non à la culture au cours des 24 heures suivant l'admission sont liés à l'établissement de santé et à une variété de facteurs propres aux patients. Lorsque des organismes pathogènes respiratoires courants sont présents dans les cultures d'expectorations, ils ont tendance à être utilisés pour assigner le diagnostic étiologique. Un résultat de culture d'expectorations positif ne semble pas s'accompagner d'un pronostic plus favorable.
\end{abstract}

S putum Gram stain and culture are recommended as part of the evaluation of a patient presenting with signs and symptoms consistent with pneumonia (1-3). This recommendation has been qualified to include only specimens that have been carefully procured, that include lower airway secretions (assessed by microscopic screening [2]), and that have been obtained before antibiotic administration (3).

For routine practice, the information obtained from sputum examination is limited. The low sensitivity of the sputum culture in the etiological diagnosis of community-acquired pneumonia (CAP) is exemplified by the finding that only $50 \%$ of patients with bacteremic pneumococcal pneumonia have Streptococcus pneumoniae in the sputum (4). Sputum culture specificity is relatively poor. Isolation of a respiratory pathogen from sputum may represent colonization of the upper airway rather than infection in the lung (5). For these reasons, some investigators choose not to use sputum culture results when defining the etiology of pneumonia in a research setting (5). There are, however, several theoretical advantages to doing a Gram stain and sputum culture. A Gram stain performed on a high quality sputum specimen, greater than 25 polymorphonuclear leucocytes/low power field (LPF) and fewer than 10 squamous epithelial cells/LPF, showing predominately Gram-positive diplococci, should suggest a diagnosis of pneumococcal pneumonia and guide therapy. In the current era of penicillinresistant pneumococci, it is important to perform sputum cultures to detect penicillin-resistant $S$ pneumoniae. This information is valuable in managing an individual patient, but it also provides valuable epidemiological information that may influence the management of other patients with CAP. Finally, a negative sputum culture in a patient who did not receive antibiotic therapy before the collection of the specimen suggests that the pneumonia may be due to an 'atypical pathogen' such as Mycoplasma pneumoniae, Legionella pneumophila or Chlamydia pneumoniae.

The objectives of the present study were to document the use of sputum Gram stain and culture results as part of the current management of CAP at four medical centres in two countries, to determine whether there were intersite differences in the procurement of specimens and to document the frequency of recovery of respiratory pathogens. We also wished to determine the factors that were associated with having a sputum sample processed for culture within $24 \mathrm{~h}$ of admission. To achieve these objectives, the percentage of patients who had sputum specimens processed for culture within 24 and 48 h of admission was determined. In addition, other areas studied included how often sputum culture results were used by the investigator to assign an etiological diagnosis, and whether a positive sputum culture was associated with a more favourable outcome versus not having a sputum culture to guide therapy.

\section{PATIENTS AND METHODS}

In-patients in the Pneumonia Patient Outcomes Research Team (PORT) cohort study were managed at four medical institutions in three geographic locations: the University of Pittsburgh Medical Center (UPMC), a 942-bed university teaching hospital, and St Francis Medical Center (SFMC), a 427-bed community teaching hospital, both in Pittsburgh, Pennsylvania; The Massachusetts General Hospital (MGH), a 899-bed university teaching hospital in Boston, Massachusetts; and The Victoria General Hospital (VGH), a 637-bed university teaching hospital in Halifax, Nova Scotia. The study was carried out from October 1991 through March 1994.

Trained nurse researchers reviewed emergency and radiology department records daily to identify potential study sub- 
jects by screening patients admitted to the participating institutions with a diagnosis of CAP. Study inclusion criteria were patient age 18 years or older, acute onset of one or more clinical symptoms suggesting pneumonia within $24 \mathrm{~h}$ of presentation, acute radiographic evidence of pneumonia within $24 \mathrm{~h}$ of presentation determined by a study site radiologist, and provision of informed consent by the patient or patient proxy. $\mathrm{Pa}$ tients were ineligible for the study if they were discharged from the hospital within the 10 days preceding presentation, were known to be human immunodeficiency virus-positive, or were previously enrolled in the cohort study. Informed consent was obtained from the patient or their guardians, and guidelines for human experimentation of the United States Department of Health and Human Services and those of the authors' institutions were followed in the conduct of this research.

Patient data collection: Following patient enrolment, trained research nurses systematically collected information at the time of presentation and throughout the clinical course of the illness. Demographic data included age, sex, ethnicity, occupation, employment status, insurance coverage and marital status.

Symptoms and the presence of comorbid conditions including history of cigarette smoking, pulmonary disease (asthma, chronic obstructive pulmonary disease or interstitial lung disease), cardiovascular disease (ischemic heart disease or congestive heart failure), cancer, diabetes mellitus and cerebrovascular disease were determined by patient reports and review of medical records. If a patient was unable to participate in a direct interview (for reasons of mental confusion or language barriers), a proxy respondent from among the patient caregivers was interviewed. Baseline physical examination findings, including heart rate, temperature, blood pressure and respiratory rate, were obtained from the medical record. Baseline laboratory data (if performed) included complete blood counts, electrolytes, serum glucose, liver function tests and arterial blood gases. Results of microbiological testing (completed at the discretion of attending physicians) were recorded (sputum Gram stain and culture, blood cultures, pleural fluid cultures and serological tests). Tests were not counted if they were ordered but not performed.

Specimen collection and microbiological methods: Sputum specimens were collected according to the methods in place at each site. These specimens were mostly expectorated sputa. Rarely (fewer than 1\%) were specimens induced sputum or respiratory secretions collected at the time of bronchoscopy (in four patients). Sputum Gram stain, culture, and identification and the reporting of micro-organisms were carried out in accordance with pre-existing protocols at each study site. At each site, laboratory protocols specified that sputum be processed for culture if there were 25 or more white blood cells and fewer than 10 squamous epithelial cells per low power field (10X magnification). The Quelling test was not performed. The reporting protocol at the UPMC differed from the other sites in that components of normal flora were reported at this site.

Definitions: Within $24 \mathrm{~h}$ was defined as the day of admission, and this included time spent in the emergency room. Within $48 \mathrm{~h}$ was the day of admission plus the next day.

Determination of etiology of pneumonia: For purposes of this study, etiology was assigned by a committee consisting of five investigators in the PORT cohort study (MF, DS, WK, CC, TM). Two investigators reviewed the results of all microbiological tests on each patient and used rules established before the study to assign etiology. If there was disagreement as to the etiology, the case was presented to the entire committee where a majority decision ruled.

All pathogens isolated from blood or pleural fluid cultures were judged to be the definite cause of the pneumonia. Isolates from sputum were categorized as the presumptive cause of the pneumonia (6) if there was one of the following:

heavy or moderate growth of a predominant bacterial pathogen;

light growth of a pathogen in which the sputum Gram stain revealed a bacterium compatible with the culture results;

bacterium isolated on multiple sputum cultures within three days of admission; and

in the case of multiple potential bacterial pathogens growing in equal magnitude, if there was a predominant organism seen on Gram stain that was compatible with one of these isolates, that organism was designated as the presumptive agent. If Gram stain revealed the presence of multiple organisms consistent with those isolated on culture, multiple pathogens were considered to be the presumptive etiology.

Medical outcomes: To determine whether a positive sputum culture favourably affected the outcome of patients with CAP, 30- and 90-day mortality rates, length of stay, median number of complications, median number of intravenous antibiotics and median duration of antibiotic therapy were compared for 861 patients who did not have a sputum specimen processed within $24 \mathrm{~h}$ of admission with the 343 patients who had a positive sputum culture with $24 \mathrm{~h}$ of admission.

Statistical analysis: Data analyses were performed using StatView version 4.02 (Abacus Concepts Inc, California) and Stata version 3.00 (Computing Resource Center, California). The significance of proportional differences between nominal variables was determined using the ${ }^{2}$ test, and differences between continuous variables were determined using Students $t$ test. A two-tailed $\mathrm{P}<0.05$ was used to define statistical significance. The significance of differences of medians of skewed variables (length of stay, number of antibiotics and number of complications) was assessed using a Wilcoxon signed rank test. To minimize the confounding of hospital site in length of stay and complications, analyses were stratified by hospital site.

To determine the factors that were associated with obtaining a sputum sample for culture, patients who had a sputum specimen processed within $24 \mathrm{~h}$ of presentation were compared with those who did not have a sputum sample processed during the first week in hospital. Factors significant in univariate analysis at $\mathrm{P}<0.05$ were entered into a multivariate stepwise logistic regression analysis. 
TABLE 1

Results of initial sputum cultures and number of patients with sputum Gram stains, carried out within 24 and $48 \mathrm{~h}$ of admission at four sites

\begin{tabular}{|c|c|c|c|c|c|c|c|c|c|c|}
\hline Characteristic & \multicolumn{2}{|c|}{ UPMC } & \multicolumn{2}{|c|}{ SFMC } & \multicolumn{2}{|c|}{ MGH } & \multicolumn{2}{|c|}{ VGH } & \multicolumn{2}{|c|}{ Total } \\
\hline $\begin{array}{l}\text { Number of patients } \\
\text { (\% of total) }\end{array}$ & \multicolumn{2}{|c|}{$331(24.7)$} & \multicolumn{2}{|c|}{ 111(8.30) } & \multicolumn{2}{|c|}{$536(40.0)$} & \multicolumn{2}{|c|}{$361(27)$} & \multicolumn{2}{|c|}{$1339(100)$} \\
\hline Time & $24 \mathrm{~h}$ & $48 \mathrm{~h}$ & $24 h^{* *}$ & $48 \mathrm{~h}$ & $24 \mathrm{~h}^{*}$ & $48 \mathrm{~h}$ & $24 h^{* *}$ & $48 \mathrm{~h}$ & $24 \mathrm{~h}$ & $48 \mathrm{~h}$ \\
\hline $\begin{array}{l}\text { Number }(\%) \text { of } \\
\text { patients with } \\
\text { sputum cultured within } \\
\text { indicated time }\end{array}$ & $125(37.8)$ & $178(53.8)$ & $25(22.5)$ & $50(450$ & $269(50.2)$ & $384(71.6)$ & $59(16.3)$ & $101(28.0)$ & $478(35.7)$ & $713(53.2)$ \\
\hline $\begin{array}{l}\text { Number (\%) of patients } \\
\text { with sputum Gram stain } \\
\text { with indicated time }\end{array}$ & $72(21.8)$ & $108(32.6)$ & $28(25.2)$ & $53(47.8)$ & $295(55)$ & $385(71.8)$ & $59(16.3)$ & $103(28.5)$ & $454(33.9)$ & $649(48.5)$ \\
\hline \multicolumn{11}{|l|}{ Number (\%) specimens with } \\
\hline Negative culture & $11(8.8)$ & $17(9.6)$ & $11(44.0)$ & $16(32)$ & $9434.9)$ & $133(34.6)$ & $19(32.2)$ & $33(32.7)$ & $135(28.2)$ & 199 (27.9) \\
\hline One species & $21(16.8)$ & $35(19.7)$ & $6(24.0)$ & $24(48)$ & $101(37.5)$ & $146(57.0)$ & $28(47.5)$ & $51(49.5)$ & $156(32.6)$ & 256 (35.9) \\
\hline $\begin{array}{l}\text { Two or more } \\
\text { species isolated }\end{array}$ & $93(74.4)$ & $126(70.8)$ & $8(32.0)$ & $10(20)$ & 74 (27.5) & $105(27.3)$ & $12(20.3)$ & 17 (16.8) & $187(39.1)$ & $258(36.2)$ \\
\hline $\begin{array}{l}\text { Mean number of species } \\
\text { reported per positive } \\
\text { culture specimen }\end{array}$ & 2.4 & 2.3 & 1.9 & 1.4 & 1.6 & 1.5 & 1.4 & 1.3 & 1.9 & 1.7 \\
\hline
\end{tabular}

*P<0.001 compared with University of Pittsburgh Medical Center (UPMC), St Francis Medical Center (SFMC), Victoria General Hospital (VGH); **P is not significant. MGH Massachusetts General Hospital

\section{RESULTS}

The study population consisted of 1339 patients hospitalized for management of CAP. The mean age was 64.3 years; $58.7 \%$ were 65 years of age or older. One hundred and eightyfour patients (13.8\%) were from a nursing home; 640 (47.7\%) were female, and $85.7 \%$ were admitted from an emergency department.

Table 1 shows the number of patients at each site and the results of the initial sputum culture carried out within 24 and $48 \mathrm{~h}$ of admission. Overall, approximately one-third of patients had a specimen processed for culture within $24 \mathrm{~h}$ of admission. Just over half the patients (53.2\%) had a sputum specimen processed for culture within $48 \mathrm{~h}$ of admission (Table 1). The percentage of patients with a sputum specimen processed for culture within $24 \mathrm{~h}$ ranged from $16.3 \%$ at VGH to 50.2 at $\mathrm{MGH}(\mathrm{P}<0.01)$. UPMC differed from the other three sites in that only $8.8 \%$ of the specimens were reported as culture negative compared with $32.2 \%$ to $44.0 \%(\mathrm{P}<0.01)$ at the other sites. Furthermore, UPMC reported $74.4 \%$ of all sputum specimens processed for culture as having multiple micro-organisms isolated. At the other sites, multiple species were reported in $20.3 \%$ to $32.0 \%$ of the specimens. The reason for these differences is apparent from an examination of Table 2 . It is evident that the following components of normal respiratory flora coagulase-negative staphylococci, micrococcus species, Neisseria species and viridans streptococci - accounted for $51.1 \%$ of all isolates at UPMC. In contrast, these isolates were not reported at the other three sites. Seventy-one per cent (341 of 478 ) of the patients who had a sputum specimen processed within $24 \mathrm{~h}$ of admission were not receiving antibiotic therapy when the specimen was collected. The percentage of patients not receiving antibiotics when specimens were collected at each site were UPMC 73\%, SFMC 96\%, MGH 69\% and VGH $69 \%$.
$S$ pneumoniae was the most commonly recovered respiratory pathogen, accounting for $12.4 \%$ of all isolates, from sputum samples processed with $24 \mathrm{~h}$ of admission (Table 2 ). This was followed by Haemophilus influenzae at $11.1 \%$ and $S$ aureus at $7 \%$. Aerobic Gram-negative bacilli (AGNR) as a group accounted for 109 of 633 (17.2\%) of the isolates. Klebsiella pneumoniae was the AGNR most commonly isolated followed by Pseudomonas aeruginosa and Escherichia coli. Moraxella catarrhalis was isolated at MGH only, where it accounted for $4.4 \%$ of the total isolates (Table 2). The rate of isolation of $S$ pneumoniae was higher at MGH than at the other sites $(\mathrm{P}<0.0001)$. The rate at which $S$ pneumoniae was recovered from sputum samples obtained before antibiotic therapy in 64 of 341 (18.7\%) patients was not significantly different from the rate obtained while the patients were receiving antibiotic therapy, 20 of 137 (14.6\%).

Table 3 shows the results of the Gram stains on 407 initial sputum specimens submitted within $24 \mathrm{~h}$ of admission. None of the specimens processed at VGH had excess (defined as 10 or greater, moderate or abundant/LPF) squamous epithelial cells (SEC), whereas $11.1 \%$ of the samples at MGH and $13.8 \%$ of those at UPMC had excessive SECs. Almost half the specimens at the American sites had many polymorphonuclear leukocytes (PMNs), while only $3.6 \%$ of the specimens at VGH had many PMNs. There were major differences in Gram stain reporting patterns among the sites (Table 3). In particular, half the smears at MGH were reported as having Gram-positive diplococci compared with only $1.8 \%$ of those in VGH. Streptococcus pneumoniae was recovered from seven $(12.7 \%)$ of the sputum specimens processed at VGH compared with 53 $(19.3 \%)$ of the sputum specimens processed (within $24 \mathrm{~h}$ of admission) at MGH (data not shown). Gram-negative rods were reported on Gram stain of $72.7 \%$ of the specimens processed 
TABLE 2

Results of initial sputum specimens processed for culture within $24 \mathrm{~h}$ of admission at four sites

\begin{tabular}{|c|c|c|c|c|c|}
\hline & UPMC $(n=125)$ & SFMC $(n=25)$ & MGH $(n=296)$ & VGH $(n=59)$ & Total $(n=478)$ \\
\hline Number of isolates & 278 & 26 & 274 & 55 & 633 \\
\hline \multicolumn{6}{|l|}{ Micro-organism isolated, number (\%) } \\
\hline Streptococcus pneumoniae & $15(5.4)$ & $3(11.5)$ & $53(19.3)$ & $8(14.5)$ & $79(12.4)$ \\
\hline Viridans streptococci* & $73(26.3)$ & 0 & 0 & 0 & $73(11.5)$ \\
\hline Haemophilus influenzae & $12(4.3)$ & $2(8)$ & $43(15.7)$ & $13(23.6)$ & $70(11.1)$ \\
\hline Neisseria species, unspecified* & $48(17.3)$ & 0 & 0 & 0 & $48(7.6)$ \\
\hline Staphylococcus aureus & $2(0.7)$ & $2(7.7)$ & $33(12.0)$ & $7(12.7)$ & $44(7.0)$ \\
\hline Klebsiella pneumoniae & $8(2.9)$ & $1(3.8)$ & $11(4.0)$ & $2(3.6)$ & $22(3.5)$ \\
\hline Pseudomonas aeruginosa & $7(2.5)$ & $1(3.8)$ & $11(4.0)$ & $3(5.5)$ & $22(3.5)$ \\
\hline Candida albicans* & $17(6.1)$ & $1(3.8)$ & 0 & 0 & $18(2.8)$ \\
\hline Escherichia coli & $3(1.1)$ & 0 & $10(3.6)$ & $3(5.5)$ & $16(2.5)$ \\
\hline Haemophilus parainfluenzae & $13(4.7)$ & $1(3.8)$ & $2(0.7)$ & 0 & $16(2.5)$ \\
\hline Micrococcus species* & $18(6.5)$ & 0 & 0 & 0 & $18(2.8)$ \\
\hline Moraxella catarrhalis & 0 & 0 & $12(4.4)$ & 0 & $12(1.9)$ \\
\hline
\end{tabular}

An additional 21 species of micro-organisms were isolated but each constituted less than $1.6 \%$ of the total number of isolates. ${ }^{*}$ Normal flora

TABLE 3

Results of Gram stain completed on sputum specimens (one per patient) submitted within $24 \mathrm{~h}$ after admission to hospital

\begin{tabular}{|c|c|c|c|c|c|}
\hline & UPMC & SFMC & MGH & VGH & Total \\
\hline $\begin{array}{l}\text { Number (\%) sputum specimens with Gram } \\
\text { stain/Number processed for culture }\end{array}$ & $65 / 125(52.0)$ & $25 / 25(100)$ & $262 / 269(97.4)$ & $55 / 59(93.2)$ & $407 / 478(85.1)$ \\
\hline \multicolumn{6}{|l|}{ Polymorphonuclear leukocytes } \\
\hline None; $<1$; scant & $31(47.7)$ & $0(0)$ & $81(30.9)$ & $15(27.3)$ & $127(31.2)$ \\
\hline 25 or more moderate; abundant & $23(35.4)$ & $15(60)$ & $126(48.1)$ & $2(3.6)$ & $166(40.8)$ \\
\hline $\begin{array}{l}\text { Squamous epithelial cells (none; } 10 \text { or less, } \\
\text { scant) }\end{array}$ & $15(23.1)$ & $0(0)$ & $107(40.8)$ & $34(61.8)$ & $156(38.3)$ \\
\hline \multicolumn{6}{|l|}{ Organism identified } \\
\hline None & $2(3.1)$ & $1(4.0)$ & $9(3.4)$ & $23(41.8)$ & $35(8.6)$ \\
\hline Predominant & $3(4.6)$ & $0(0)$ & $2(0.8)$ & $27(49.1)$ & $32(7.9)$ \\
\hline Mixed & $3(4.6)$ & $0(0)$ & $23(8.8)$ & $3(5.5)$ & $29(7.1)$ \\
\hline Gram-positive diplococci & $12(18.5)$ & $8(32.0)$ & $133(50.8)$ & $1(1.8)$ & $154(37.8)$ \\
\hline Gram-positive cocci in chains & $16(24.6)$ & $12(15.4)$ & $43(55.1)$ & $7(9.0)$ & $78(19.2)$ \\
\hline Gram-positive cocci in clusters & $14(21.5)$ & $11(44.0)$ & $35(13.4)$ & $1(1.8)$ & $61(15.0)$ \\
\hline Gram-negative rods & $25(38.5)$ & $15(60.0)$ & $50(19.1)$ & $40(72.7)$ & $130(31.9)$ \\
\hline House staff performed Gram stain & $24(36.9)$ & $0(0.0)$ & $129(49.2)$ & $1(1.8)$ & $154(37.8)$ \\
\hline
\end{tabular}

for culture at the Halifax site, while only $19.1 \%$ of those at the Boston site were reported as having Gram-negative rods. Table 3 also shows that house staff rarely performed Gram stains on sputum samples at SFMC and VGH, while at UPMC and MGH $36.9 \%$ and $49.2 \%$ of the sputum specimens respectively were Gram stained by house staff.

Investigators assigned an etiological diagnosis for 397 of the 1339 (29.6\%) patients. Sputum culture results were frequently used to assign an etiological diagnosis (Table 4). Indeed for the 397 patients with an etiological diagnosis (Table 4), 265 (66.7\%) were assigned on the basis of sputum culture results only. Table 4 shows that $S$ pneumoniae and $H$ influenzae, if isolated from the sputum, were usually accepted by the investigators as causing the pneumonia. Indeed, $97.7 \%$ and $88.4 \%$ of these organisms, respectively, isolated from sputum were used to assign an etiological diagnosis. In contrast, sputum isolates of Proteus mirabilis, Serratia marce- sans and $E$ coli were infrequently used (25\% to $28 \%$ of the time) to assign an etiology while the use of isolates of $P$ aeruginosa, $K$ pneumoniae, Enterobacter cloacae, $H$ parainfluenzae and $M$ catarrhalis was intermediate (47.3\% to $67.2 \%$ ) between that of the other two groups.

Sixty-five of the 397 (16.4\%) patients with pneumonia of known etiology had multiple, ie, two or more, etiological agents identified. Five patients had three microbial agents implicated, and three had four agents. Sputum isolates were used to assign an etiology in 57 of the 65 patients $(87.7 \%)$. Of the remaining eight patients, four had no sputum sample processed (these four had diagnoses made by pleural fluid culture or by serology). Specimens obtained by bronchoscopy were used to make a diagnosis for the other four patients. Thirty-four of the 61 (55.7\%) patients with multiple etiological agents assigned on the basis of sputum culture had all of the assigned agents present in the first sputum sample processed 
TABLE 4

Contribution of sputum culture results to etiology of pneumonia

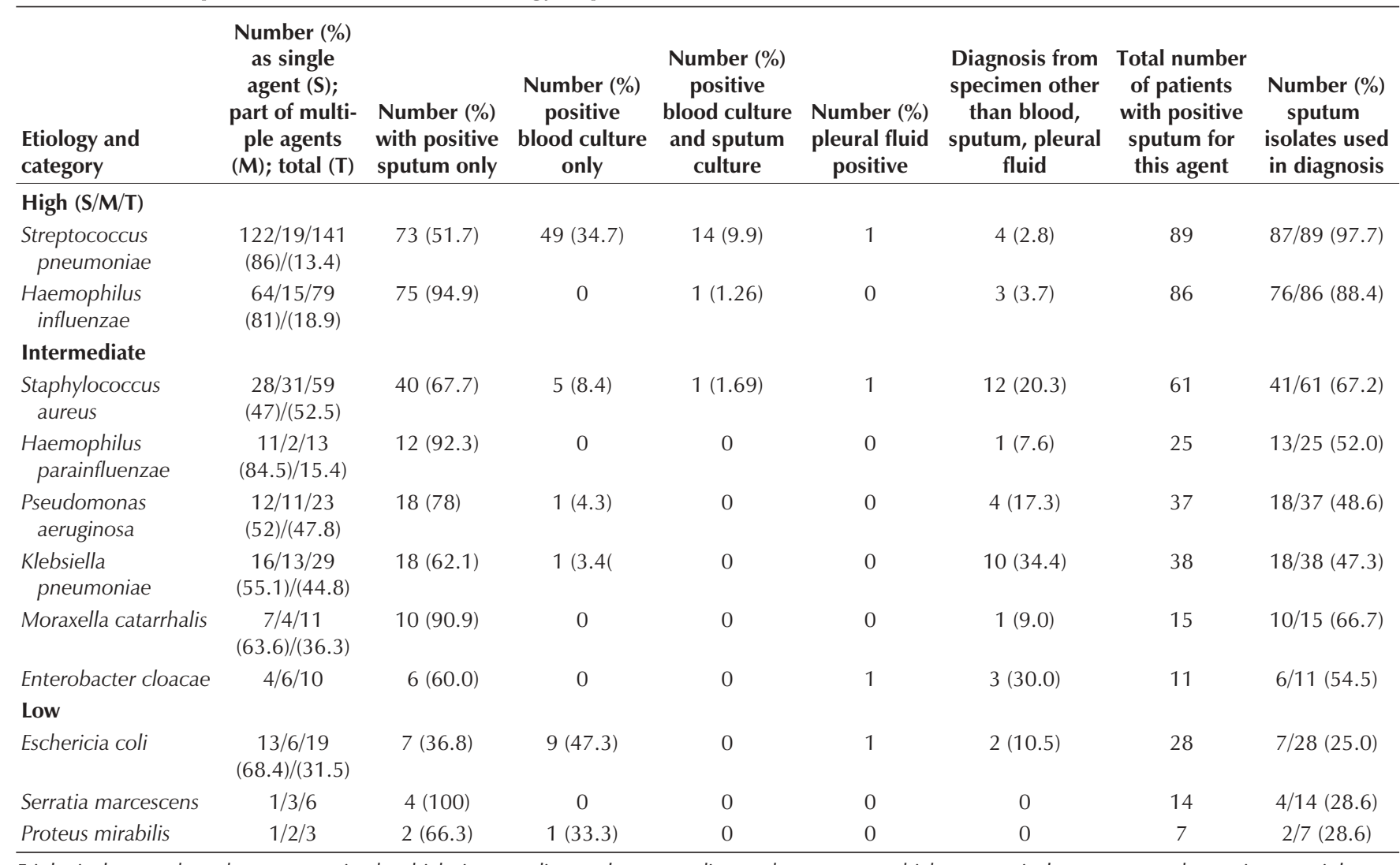

Etiological agents have been categorized as high, intermediate or low according to the extent to which sputum isolates were used to assign an etiology

for culture. An additional 18 had at least one of the assigned agents present in the first specimen. The 65 patients with polymicrobial etiology had a total of 139 agents implicated. $S$ aureus was most common accounting for 31 cases; $S$ pneumoniae was next at 19 , followed by $H$ influenzae at 15 , $K$ pneumoniae at 13, $P$ aeruginosa at 11 and group B streptococcus at five. The most common combinations were $S$ aureus and $S$ pneumoniae in eight specimens, $S$ pneumoniae and $H$ influenzae in six, $S$ aureus and group B streptococcus in four, and $P$ aeruginosa and other AGNR in eight. In one instance, $P$ aeruginosa was found in combination with $H$ influenzae.

Multivariate analysis of factors that predicted sputum culture that were significant on univariate analysis revealed that MGH (odds ratio $20.6 ; 95 \% \mathrm{CI} 11.4$ to 37.3 ) as the site of care was the most important factor in whether a sputum was processed for culture during the first $24 \mathrm{~h}$ of hospitalization (Table 5). It is also evident that some patient factors, such as amount of sputum production and chronic obstructive pulmonary disease, increased the patient's likelihood of having sputum processed for culture, while neutropenia, do not resuscitate status and female sex had a negative effect.

When patients who had a positive sputum culture were compared with those who had no specimen processed for culture, no difference in outcomes were found 30- and 90-day mortality, length of stay, number of complications or number and duration of antibiotic therapy (Table 6).

\section{DISCUSSION}

This study demonstrates the current use of the sputum culture and Gram stain in the management of patients with CAP requiring hospitalization. The first important observation is that only just over one-third of the 1339 patients in this multicentre study had a sputum specimen processed for culture within $24 \mathrm{~h}$ of admission. Moreover, during this period only $25 \%$ of the total patient population had a sputum culture "positive for a pathogen". The rate of sputum submission varied by institution: it was lowest at VGH ( $28 \%$ within $48 \mathrm{~h}$ of admission) and highest at MGH (72\%). Multivariate analysis showed that site of care (a patient hospitalized at MGH was 20 times more likely to have a sputum specimen processed for culture within $24 \mathrm{~h}$ of admission than a patient hospitalized at VGH) was the most important determinant in having a sputum processed for culture within $24 \mathrm{~h}$ of presentation. It was also apparent that patient factors had both positive (current sputum production, chronic obstructive pulmonary disease and quantity of sputum production) and negative (do not resuscitate status, neutropenia and female sex) influences on the frequency that sputum was submitted for culture. Local practice variation in the use of sputum culture to diagnose pneumonia is exemplified by Bates et al (5) who, in a study of 198 patients with 204 episodes of pneumonia (154 were communityacquired) at Little Rock, Arkansas, did not use sputum Gram stain and culture data to assign an etiological diagnosis. They 
TABLE 5

Independent predictors of having a sputum specimen processed for culture within $24 \mathrm{~h}$ of admission by stepwise logistic regression analysis of factors significant on univariate analysis for 633 patient respondents

\begin{tabular}{lccc}
\hline & Odds ratio & $\mathbf{9 5 \%} \mathbf{C l}$ & P \\
\hline Massachusetts General Hospital as site of care & 20.6 & $11.4-37.3$ & 0.0001 \\
UPMC and SFMC as sites of care & 3.4 & $2.0-5.9$ & 0.0001 \\
Chronic obstructive pulmonary disease & 1.8 & $1.1-2.9$ & 0.02 \\
Current sputum production & 4.9 & $2.8-8.4$ & 0.0001 \\
Moderate or large amount of sputum & 2.7 & $1.5-4.5$ & 0.004 \\
Neutropenia & 0.06 & $0.005-0.9$ & 0.04 \\
Female sex & 0.4 & $0.3-0.7$ & 0.0006 \\
Do not resuscitate status & 0.36 & $0.1-0.9$ & 0.04 \\
\hline
\end{tabular}

UPMC University of Pittsburgh Medical Center, SFMC St Francis Medical Center

TABLE 6

Outcomes of treatment of community-acquired pneumonia according to results of sputum cultures processed within $24 \mathrm{~h}$ of admission and for patients who did not have a sputum specimen submitted for culture within this period

\begin{tabular}{|c|c|c|c|}
\hline & $\begin{array}{l}\text { Sputum processed for } \\
\text { culture positive } \\
(n=343)\end{array}$ & $\begin{array}{l}\text { Sputum not processed } \\
\text { for culture } \\
(\mathbf{n}=\mathbf{8 6 1})\end{array}$ & $\mathbf{P}$ \\
\hline 30-day mortality & $25(7.3 \%)$ & $77(8.9 \%)$ & $0.86^{*}$ \\
\hline 90-day mortality & $43(12.5 \%)$ & $142(16.5 \%)$ & 0.19 \\
\hline Median length of stay (days) & 7 & 8 & 0.22 \\
\hline Median number of complications during hospital stay & 1 & 2 & ND \\
\hline Median number of intravenous antibiotics during hospital stay & 2 & 2 & 0.10 \\
\hline Median duration of antibiotic therapy during hospital stay (days) & 6 & 6 & 0.57 \\
\hline
\end{tabular}

*All P are for comparisons stratified by hospital (Victoria General Hospital, Massachusetts General Hospital, St Francis Medical Center, University of Pittsburgh Medical Center). ND Not done

did this because they used very strict criteria and they "did not accept Gram stain and culture data from expectorated sputum" (5). Because of our study design, we did not collect data on specimens submitted but not processed for culture. It is possible that the numbers of specimens submitted (including both processed and rejected samples) may have not shown as much intersite variation.

One of the unexpected findings from our study was the way sputum culture results were reported at the various sites. At UPMC, components of normal respiratory flora (viridans streptococci, Neisseria species, Micrococcus species) accounted for a large portion of the isolates. This information is of no value to the clinician and in some instances may be misleading. This is an example of a local practice variation because the laboratory protocols for processing, plating and reporting of sputum specimens at all participating sites were essentially identical.

About one-third of the sputum processed for culture were inadequate based on Gram stain data in that too few PMNs or excess epithelial cells were present (2). VGH processed no specimens with too many SECs, while these constituted $12.5 \%$ of the specimens at the other three sites. Streptococcus pneumoniae was isolated from $14 \%$ of 619 sputum specimens while Gram-positive diplococci were reported on Gram stain in $32.1 \%$ of these samples. A meta-analysis of the sensitivity and specificity of the sputum Gram stain in community-acquired pneumococcal pneumonia found that the sensitivity ranged from $15 \%$ to $100 \%$ and specificity from $11 \%$ to $100 \%$. Test characteristics varied markedly among studies and appeared to be re- lated partly to the test interpreter (7). Rein et al (8) considered a sputum Gram stain to be positive for pneumococci if more than 10 lancet-shaped diplococci were seen per oil immersion field. In their study this criterion was met in 18 of 29 (62\%) specimens from which $S$ pneumoniae was isolated. Others (9) have found that experience is important in the interpretation of the Gram stain-pneumococci tend to be overdiagnosed and $H$ influenzae underdiagnosed by house staff when interpreting Gram stains. House staff performed a Gram stain on $40 \%$ of the sputum samples processed for culture at MGH and $28.4 \%$ of those at UPMC. In contrast, only $1 \%$ (one specimen) was Gram stained by house staff at VGH, and none were examined by house staff at SFMC. These differences reflect hospital policies in that microscopes and reagents are not available to house staff at VGH to perform Gram stain. Gram stain can be used to guide the microbiology laboratory staff to look for specific micro-organisms (using colony morphology) on the culture plates. Heineman et al (10) used this approach and found that routine processing of specimens without reference to the Gram stain missed $61 \%$ of $S$ pneunioniae, $23 \%$ of Haemophilus species and $44 \%$ of yeast that were found by Gram stain-directed culture.

$M$ catarrhalis was isolated only at MGH. This microorganism is infrequently carried in the upper respiratory tract of adults (5.4\% of adults), and its presence in sputum is rarely due to oronasopharyngeal contamination (11). In studies carried out at Johnson City Veterans Administration Medical Center (Tennesee), $M$ catarrhalis was recovered from $2.7 \%$ of all 
respiratory specimens submitted over a 42-month period and was the second most commonly isolated respiratory pathogen. $M$ catarrhalis is sometimes overlooked unless culture processing is guided by correct Gram stain interpretation $(12,13)$. The rate of isolation of $S$ pneumoniae was higher at MGH than at the other three sites. The reason for this finding is not evident from our study.

Sixteen per cent of our patients with pneumonia of known etiology had multiple pathogens implicated. Lieberman et al (14) studied 346 patients with CAP; 133 (38.4\%) had more than one causal agent; and indeed $10.7 \%$ had three or more agents implicated, although these authors implicated many of the multiple agents as a result of comprehensive aerological studies.

Because the majority of patients with CAP do not have sputum specimens submitted or the specimens are not submitted early enough in the course of their hospital stay to be useful in management, the question arises whether sputum specimens should be obtained at all. Woodhead et al (15) studied 122 consecutive patients with pneumonia admitted to a district hospital in England. Eighty-one per cent had blood cultures performed, $45 \%$ had sputum examined and $28 \%$ had aerological testing performed. The results of these microbiological tests directed a change in antibiotic therapy in only $8 \%$ of

\section{REFERENCES}

1. Neu HC. Pneumonia. In: Stein JH, Hutton JJ, Kohler PO, et al, eds. Internal Medicine, 4th edn. St Louis: Mosby, 1994:1868-77.

2. Murray PR, Washington JAJ. Microscopic and bacteriologic analysis of expectorated sputum. Mayo Clin Proc 1979;50:339-44

3. Bartlett JG, Mundy LM. Community-acquired pneumonia. N Engl J Med 1995;333:1618-24.

4. Barret-Connor E. The nonvalue of sputum culture in the diagnosis of pneumococcal pneumonia. Am Rev Respir Dis 1971;103:845-8

5. Bates JH, Campbell GD, Barron AL, et al. Microbial etiology of acute pneumonia in hospitalized patients. Chest 1992;101:1005-12.

6. Fang G-D, Fine M, Orloff J, et al. New and emerging etiologies for community-acquired pneumonia with implications for therapy. A prospective study of 359 cases. Medicine 1990;69:307-31.

7. Reed WW, Byrd GS, Gates RH Jr, Howard RS, Weaver MJ. Sputum Gram's stain in community-acquired pneumococcal pneumonia. A meta-analysis. West J Med 1996;165:197-204.

8. Rein MF, Gwaltney JM, O'Brien WM, Jennings RH, Mandell G. Accuracy of Gram's stain in identifying pneumococci in sputum. JAMA 1978;239:2671-3.

9. Fine MJ, Orloff JJ, Rihs JD, et al. Evaluation of housestaff physicians' preparation and interpretation of sputum Gram stains for community-acquired pneumonia. J Gen Intern Med 1991;6:189-98. cases. They concluded that routine microbiological investigation of all adults admitted to hospital with CAP is unhelpful and probably unnecessary. Chalasani and coworkers (16) found that $6.6 \%$ of 517 adults with CAP were bacteremic, and antibiotic therapy was changed for seven of the 34 patients with positive blood cultures. These workers concluded that blood cultures may have limited utility and questionable cost effectiveness in the management of CAP. From our data, one can argue that sputum examination contributes little towards the management of CAP. However, our study did not address the issue of penicillin-resistant $S$ pneumoniae, which at present is a reason to promote culturing sputum in all patients requiring hospital admission for treatment of pneumonia.

ACKNOWLEDGEMENTS: This research was supported by a grant-inaid from the Agency for Health Care Policy and Research (Grant RO1HS-06468). Ms Taylor was supported by a student summer research fellowship, Faculty of Medicine, Dalhousie University, Halifax, Nova Scotia. Dr Fine was supported in part as a Robert Wood Johnson Foundation Generalist Physician Faculty Scholar. We thank Dr John LeBlanc for advice regarding data analysis and clinical research assistants Donna Polenick RN (Pittsburgh); Mary Ungaro RN and Leila Haddad AB (Boston); Rhonda Grandy RN, Jackie Cunning RN, Dawn Menon GN and Linda Kraft RN (Halifax).

10. Heineman HS, Chawla JK, Lofton WM. Misinformation from sputum cultures without microscopic examination. J Clin Microbiol 1977;6:518-27.

11. Vaneechoutte M, Verschraegen G, Claeys G, Weise B, Van den Abeele AM. Respiratory tract carriage rates of Moraxella (Branhamella) catarrhalis in adults and interpretation of the isolation of $M$ catarrhalis from sputum. J Clin Microbiol 1990;28:2674-80.

12. Sarubbi FA, Myers JW, Williams JJ, Shell CG. Respiratory infections caused by Branhamella catarrhalis. Am J Med 1990;88(Suppl 5A):95-145.

13. Ainsworth SM, Nagy SB, Morgan LA, Miller GR, Perry JL. Interpretation of Gram-stained sputum containing Moraxella (Branhamella) catarrhalis. J Clin Microbiol 1990;28:2559-60.

14. Lieberman D, Schlaeffer F, Boldur I, et al. Multiple pathogens in adult patients admitted with community-acquired pneumonia: a one year prospective study of 346 consecutive patients. Thorax 1996;51:179-84.

15. Woodhead MA, Arrowsmith J, Chamberlin-Webber R, Wooding S, Williams I. The value of routine microbial investigation in community-acquired pneumonia. Respir Med 1991;85:313-7.

16. Chalasani NP, Valdecanas MAL, Gopal AK, McGowan JE Jr, Jurado RL. Clinical utility of blood cultures in adult patients with community-acquired pneumonia without defined underlying risks. Chest 1995;108:932-6. 


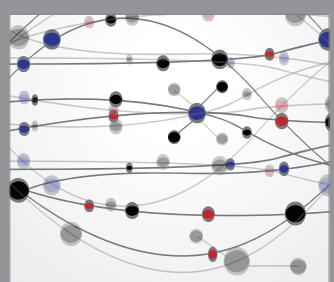

The Scientific World Journal
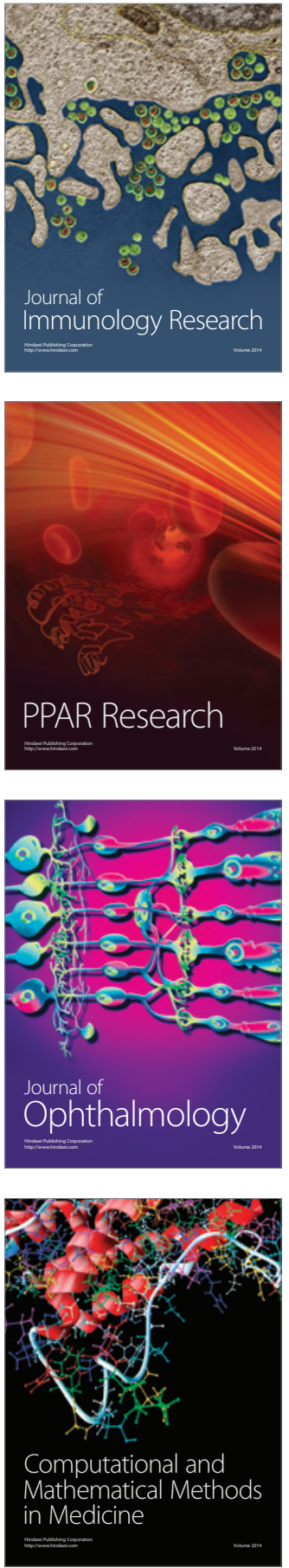

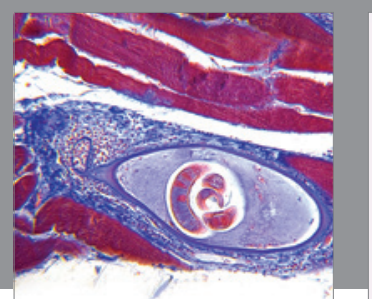

Gastroenterology Research and Practice

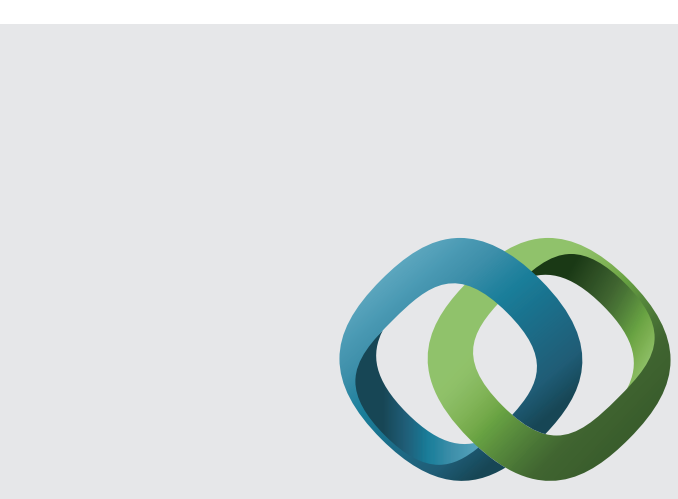

\section{Hindawi}

Submit your manuscripts at

http://www.hindawi.com
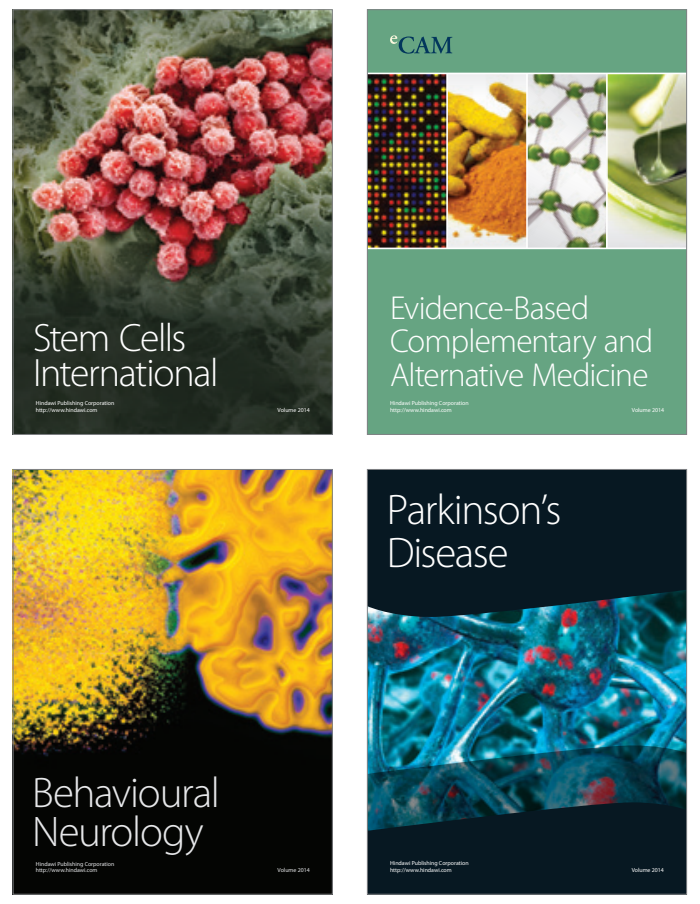
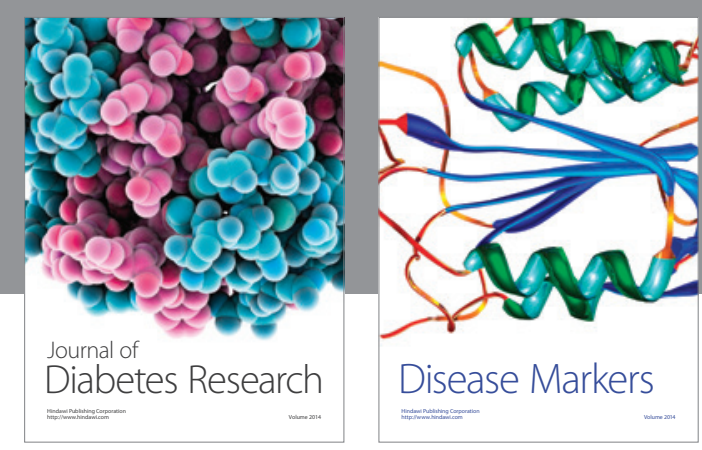

Disease Markers
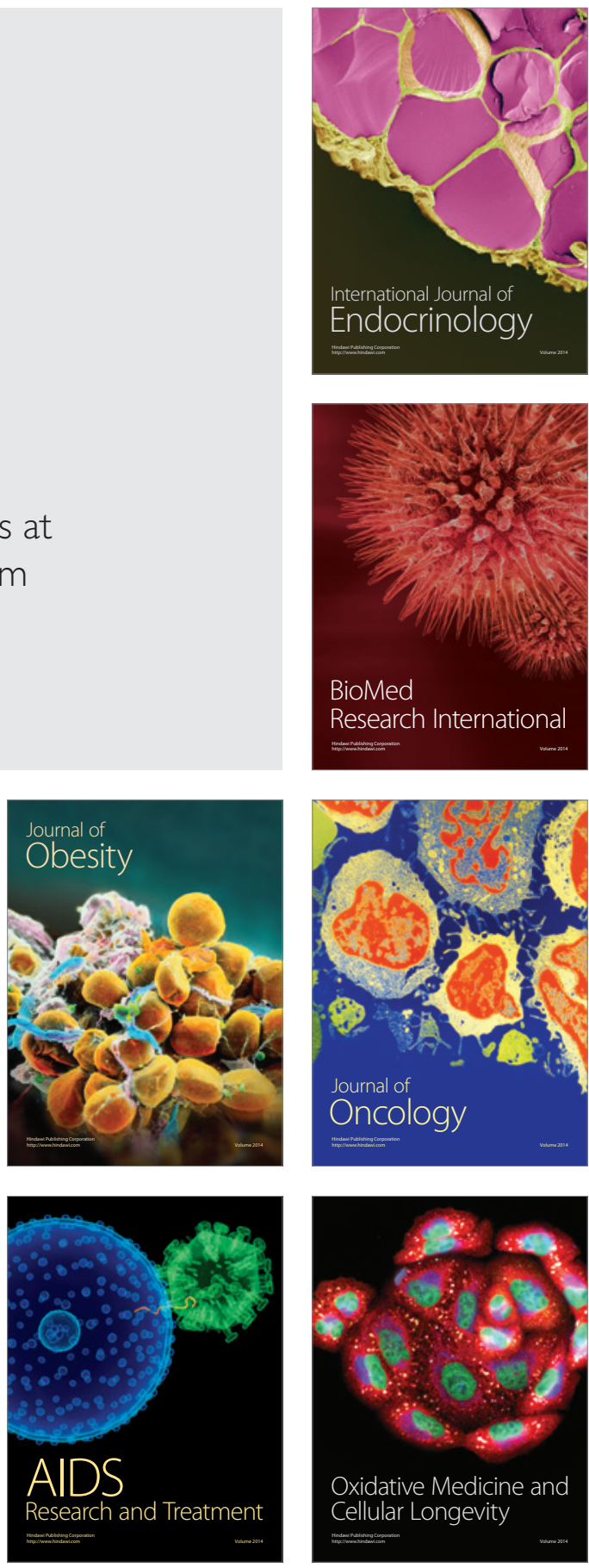